\title{
THE MEAN VALUES OF DIRICHLET $L$-FUNCTIONS AT INTEGER POINTS AND CLASS NUMBERS OF CYCLOTOMIC FIELDS
}

\section{MASANORI KATSURADA * AND KOHJI MATSUMOTO}

\section{Introduction}

Let $q$ be a positive integer, and $L(s, \chi)$ the Dirichlet $L$-function corresponding to a Dirichlet character $\chi \bmod q$. We put

$$
V_{q}=\sum_{\substack{\chi(\bmod q) \\ \chi \neq \chi_{0}}}|L(1, \chi)|^{2},
$$

where $\chi$ runs over all Dirichlet characters $\bmod q$ except for the principal character $\chi_{0}$.

At first we consider the case $q=p$ is a prime number. Let $\zeta(s)$ be the Riemann zeta-function. The classical result

$$
V_{p}=\zeta(2) p+O\left((\log p)^{2}\right)
$$

of Paley and Selberg (see Ankeny-Chowla [1]) was improved by Slavutskiĩ [7] [8], who proved

$$
V_{p}=\zeta(2) p-(\log p)^{2}+O(\log p) .
$$

Further refinements were given by Zhang. He improved the error estimate in (1.1) to $O(\log \log p)$ in [17], and then in [18], he obtained

$$
V_{p}=\zeta(2) p-(\log p)^{2}-\left(1+\zeta(2)+2 A-\int_{0}^{1} C(y)^{2} d y\right)+O\left(\frac{1}{\log p}\right)
$$

where

$$
A=\sum_{n=1}^{\infty} \frac{\log (n+1)}{n(n+1)} \quad \text { and } \quad C(y)=\sum_{n=1}^{\infty} \frac{y}{n(n+y)} .
$$

Received June 10, 1993.

* Partially supported by Grant-in-Aid for Scientific Research (No. 03740051), Ministry of Education, Science and Culture. 
In the present paper, by a method quite different from those of Slavutskir and Zhang, we will prove the following asymptotic expansion:

THEOREM 1. For any integer $N \geq 1$, we have

$$
\begin{aligned}
V_{p}= & \zeta(2) p-(\log p)^{2}+\left(\gamma_{0}^{2}-2 \gamma_{1}-3 \zeta(2)\right)-\left(\gamma_{0}^{2}-2 \gamma_{1}-2 \zeta(2)\right) p^{-1} \\
& +2\left(1-p^{-1}\right)\left\{\sum_{n=1}^{N-1}(-1)^{n} \zeta(1-n) \zeta(1+n) p^{-n}+O\left(p^{-N}\right)\right\}
\end{aligned}
$$

where the $O$-constant depends only on $N$, and the constants $\gamma_{0}$ and $\gamma_{1}$ are the Laurent expansion coefficients of $\zeta(s)$ at $s=1$ defined by

$$
\zeta(s)=\frac{1}{s-1}+\gamma_{0}+\gamma_{1}(s-1)+\cdots \cdots .
$$

In the right-hand side of (1.3) (and also throughout this paper), the sum $\sum_{n=1}^{N-1}$ is to be considered as the empty sum if $N=1$.

Remark. Comparing (1.2) with (1.3), we see

$$
1+2 A-\int_{0}^{1} C(y)^{2} d y=-\gamma_{0}^{2}+2 \gamma_{1}+2 \zeta(2)
$$

We will give a direct proof of this relation in Section 4 .

We define the Bernoulli numbers $B_{n}$ by

$$
\frac{z}{e^{z}-1}=\sum_{k=0}^{\infty} \frac{B_{k}}{k !} z^{k} \quad(|z|<2 \pi)
$$

It is well known that

$$
\zeta(-n)=(-1)^{n} \cdot \frac{B_{n+1}}{n+1}=\left\{\begin{aligned}
-\frac{1}{2} & \text { if } n=0, \\
-\frac{B_{n+1}}{n+1} & \text { if } n \text { is odd, } \\
0 & \text { if } n \text { is even }(n \geq 2)
\end{aligned}\right.
$$

(see (2.4.3) of Titchmarsh [10], noting that the definition of Bernoulli numbers is different in Titchmarsh's book). Therefore we can rewrite (1.3) to

$$
V_{p}=\zeta(2) p-(\log p)^{2}+\left(\gamma_{0}^{2}-2 \gamma_{1}-3 \zeta(2)\right)-\left(\gamma_{0}^{2}-2 \gamma_{1}-2 \zeta(2)\right) p^{-1}
$$




$$
+2\left(1-p^{-1}\right)\left\{\frac{1}{2} \zeta(2) p^{-1}-\sum_{1 \leq m \leq \frac{N-1}{2}} \frac{B_{2 m}}{2 m} \zeta(1+2 m) p^{-2 m}+O\left(p^{-N}\right)\right\}
$$

so the coefficients of the asymptotic expansion of $V_{p}$ can be written by using the values of $\zeta(s)$ at odd integers.

We can show the similar type of asymptotic expansions at any other integer points. For any integer $m(\neq 1)$ and $q(\geq 2)$, we put

$$
U_{q}(m)=\varphi(q)^{-1} \sum_{\chi(\bmod q)}|L(m, \chi)|^{2},
$$

where $\varphi(q)$ is Euler's function. Let $\Gamma(z)$ be the gamma-function, and define

$$
\left(\begin{array}{c}
z \\
n
\end{array}\right)=\frac{\Gamma(z+1)}{\Gamma(z-n+1) n !}
$$

for any complex $z$ and any non-negative integer $n$. Then,

THEOREM 2. For any integers $m$ and $N$ satisfying $2 \leq m \leq N$, we have

$$
\begin{aligned}
U_{p}(m)= & \zeta(2 m)+2 p^{1-2 m}(-1)^{m} \frac{(2 m-2) !}{((m-1) !)^{2}} \zeta(2 m-1) \\
& \cdot\left(-\log p+\sum_{h=m}^{2 m-2} \frac{1}{h}-\gamma_{0}+\frac{\zeta^{\prime}}{\zeta}(2 m-1)\right)-p^{-2 m}\left(\zeta(m)^{2}-2 S^{*}(m ; p)\right),
\end{aligned}
$$

where

$$
S^{*}(m ; k)=\sum_{\substack{0 \leq n \leq N-1 \\
n \neq m-1}}\left(\begin{array}{c}
-m \\
n
\end{array}\right) \zeta(m-n) \zeta(m+n) k^{m-n}+O\left(k^{m-N}\right) .
$$

THEOREM 3. For any integer $m \geq 0$, we have the following finite expression:

$$
\begin{aligned}
U_{p}(-m)= & \zeta(-2 m)-p^{2 m} \zeta(-m)^{2}+(-1)^{m-1} p^{1+2 m} \frac{(m !)^{2}}{(2 m+1) !} \zeta(-2 m-1) \\
& +2 \sum_{n=0}^{m}\left(\begin{array}{c}
m \\
n
\end{array}\right) \zeta(-m-n) \zeta(-m+n) p^{m-n}+p^{-1} \cdot \frac{B_{2 m+2}}{(m+1)^{2}} .
\end{aligned}
$$

We will prove Theorem 1 in Section 2, and Theorems 2 and 3 in Section 3. Also, we can show generalizations of the above theorems to the case of any composite modulus $q$, which will be discussed in the last section.

Let 


$$
f(x, z)=\frac{z e^{x z}}{e^{z}-1},
$$

and define the Bernoulli polynomials $B_{n}(x)$ as the Taylor expansion coefficients of $f(x, z)$ :

$$
f(x, z)=\sum_{n=0}^{\infty} \frac{B_{n}(x)}{n !} z^{n} \quad(|z|<2 \pi)
$$

Then it is known that, for any positive integer $q$ and any Dirichlet character $\chi \bmod q$, the relation

$$
L(-m, \chi)=-\frac{q^{m}}{m+1} \sum_{a=1}^{q} \chi(a) B_{m+1}\left(\frac{a}{q}\right)
$$

holds for any non-negative integer $m$ (see, e.g., Theorem 4.2 of Washington [13]). It should be noted that Theorem 3 can also be deduced from this formula (see Section 4).

Now let $p$ be an odd prime, and $h_{p}$ the class number of the cyclotomic exten$\operatorname{sion} \mathbf{Q}\left(e^{2 \pi i / p}\right)$ of the rational number field $\mathbf{Q}$. Then it is classically known that

$$
h_{p} R_{p}=2 p^{p / 2}(2 \pi)^{-(p-1) / 2} A_{p},
$$

where $R_{p}$ is the regulator of $\mathbf{Q}\left(e^{2 \pi i / p}\right)$ and

$$
A_{p}=\prod_{\substack{x(\bmod p) \\ \chi \neq \chi 0}}|L(1, \chi)| .
$$

By using the inequality $A_{p} \leq\left(V_{p} /(p-2)\right)^{(p-2) / 2}$ and (1.1), Slavutskir [7] [8] proved

$$
h_{p}<20\left(\frac{\pi}{12} p\right)^{(p-2) / 2}
$$

Recently, Wang [12] improved this result to

$$
h_{p}<10\left(\frac{\pi}{16} p\right)^{(p-2) / 2} \text {. }
$$

Wang's basic idea is to divide $A_{p}=A_{p}^{\prime} A_{p}^{\prime \prime}$, where

$$
A_{p}^{\prime}=\prod_{\substack{\chi(\bmod p) \\ \chi: \text { odd }}}|L(1, \chi)|, \quad A_{p}^{\prime \prime}=\prod_{\substack{\chi(\bmod p) \\ \chi: \text { even } \\ \chi \neq \chi_{0}}}|L(1, \chi)|,
$$

and treat each product separately. 
Combining our Theorem 1 with Walum's [11] result

$$
\sum_{\substack{\chi(\bmod p) \\ \chi: \text { odd }}}|L(1, \chi)|^{2}=\frac{(p-1)^{2}(p-2)}{12 p^{2}} \pi^{2},
$$

we get the asymptotic formula

$$
\begin{aligned}
\sum_{\substack { \chi(\bmod p) \\
\begin{subarray}{c}{x=\operatorname{even} \\
\chi \neq \chi_{0}{ \chi ( \operatorname { m o d } p ) \\
\begin{subarray} { c } { x = \operatorname { e v e n } \\
\chi \neq \chi _ { 0 } } }\end{subarray}}|L(1, \chi)|^{2} & =\frac{1}{2} \zeta(2) p-(\log p)^{2}+\left(\gamma_{0}^{2}-2 \gamma_{1}-\zeta(2)\right) \\
& -\left(\gamma_{0}^{2}-2 \gamma_{1}+\frac{1}{2} \zeta(2)\right) p^{-1}+\zeta(2) p^{-2} \\
& +2\left(1-p^{-1}\right)\left\{\sum_{n=1}^{N-1}(-1)^{n} \zeta(1-n) \zeta(1+n) p^{-n}+O\left(p^{-N}\right)\right\},
\end{aligned}
$$

which itself is of some interest. Applying this formula to $A_{p}^{\prime \prime}$, we can show an improvement of (1.12).

However, we do not state the result here, because we can prove a better estimate, by refining Wang's argument. In the Appendix we will prove that for any small $\varepsilon>0$,

$$
h_{p}=O\left(\left(\frac{\pi}{50} p\right)^{(p-2) / 2} \exp \left(-\frac{1-\varepsilon}{\pi^{2}}(\log p)^{2}\right)\right),
$$

where the $O$-constant depends only on $\varepsilon$.

The present paper is a continuation of the authors' previous paper [5], which is inspired by Motohashi's article [6]. Therefore, we can say that the origin of our method lies in the classical paper of Atkinson [2].

The authors would like to thank Professors Shigeki Egami and Yuji Kida for valuable suggestions, and Professor Tauno Metsänkylä for informing them of the existence of Wang's paper [12].

\section{The mean square of $L(1, \chi)$}

In this and the next section $p$ denotes an arbitrary prime number. First we quote the formula $[5,(4.7)$ and (1.11)]:

$$
\begin{aligned}
& (p-1)^{-1} \sum_{\chi(\bmod p)} L(u, \chi) L(v, \bar{\chi}) \\
& =\zeta(u+v)-p^{-u-v} \zeta(u) \zeta(v)+p^{1-u-v} \Gamma(u+v-1) \zeta(u+v-1)
\end{aligned}
$$




$$
\begin{aligned}
& \cdot\left(\frac{\Gamma(1-u)}{\Gamma(v)}+\frac{\Gamma(1-v)}{\Gamma(u)}\right) \\
& +\sum_{n=0}^{N-1} p^{-n}\left\{\left(\begin{array}{c}
-v \\
n
\end{array}\right) p^{-v} \zeta(u-n) \zeta(v+n)+\left(\begin{array}{c}
-u \\
n
\end{array}\right) p^{-u} \zeta(v-n) \zeta(u+n)\right\} \\
& +p^{-v-N} R_{N}(u, v ; p)+p^{-u-N} R_{N}(v, u ; p)
\end{aligned}
$$

for any $N \geq 1$, where

$$
\begin{aligned}
R_{N}(u, v ; k)=\frac{1}{\Gamma(u) \Gamma(v)} \int_{0}^{\infty} \frac{y^{v+N-1}}{e^{y}-1} \int_{0}^{\infty} \int_{0}^{1} \frac{(1-\tau)^{N-1}}{(N-1) !} \\
\cdot h^{(N)}\left(x+k^{-1} \tau y\right) x^{u-1} d \tau d x d y
\end{aligned}
$$

with

$$
h(z)=\frac{e^{z}}{e^{z}-1}-\frac{1}{z} .
$$

(The factor $y^{v+N+1}$ in $[5,(1.11)]$ should be read as $y^{v+N-1}$.) In [5] we remark that the integral in the right-hand side of (2.2) is convergent absolutely for $0<\Re(u)<N+1, \Re(v)>-N+1$. Hence (2.1) is valid in the region $\{(u, v) \mid 0<\Re(u)<N+1,0<\Re(v)<N+1\}$ as an identity of meromorphic functions. In particular, for any $N \geq 1,(2.1)$ is valid near $u=v=1$. We put $u=v=1+\delta$ with a small positive $\delta$ in (2.1) to obtain

$$
\begin{aligned}
& (p-1)^{-1} \sum_{\substack{x(\bmod p) \\
\chi \neq x_{0}}}|L(1+\delta, \chi)|^{2} \\
& =-(p-1)^{-1}\left(1-p^{-1-\delta}\right)^{2} \zeta(1+\delta)^{2}+\zeta(2+2 \delta)-p^{-2-2 \delta} \zeta(1+\delta)^{2} \\
& +2 p^{-1-2 \delta} \Gamma(1+2 \delta) \Gamma(-\delta) \Gamma(1+\delta)^{-1} \zeta(1+2 \delta)+2 p^{-1-\delta} \zeta(1+\delta)^{2} \\
& +2 p^{-2-2 \delta}\left\{\sum_{n=1}^{N-1}\left(\begin{array}{c}
-1-\delta \\
n
\end{array}\right) p^{1+\delta-n} \zeta(1+\delta-n) \zeta(1+\delta+n)\right. \\
& \left.+p^{1+\delta-N} R_{N}(1+\delta, 1+\delta ; p)\right\} .
\end{aligned}
$$

From (1.4) we see

$$
\zeta(1+\delta)^{2}=\delta^{-2}\left\{1+2 \gamma_{0} \delta+\left(\gamma_{0}^{2}+2 \gamma_{1}\right) \delta^{2}+O\left(\delta^{3}\right)\right\}
$$

so

$$
\begin{aligned}
& \left\{-(p-1)^{-1}\left(1-p^{-1-\delta}\right)^{2}-p^{-2-2 \delta}+2 p^{-1-\delta}\right\} \zeta(1+\delta)^{2} \\
& \quad=p^{-1} \delta^{-2}\left\{1+2\left(\gamma_{0}-\log p\right) \delta+\left(\gamma_{0}^{2}+2 \gamma_{1}+\frac{p-2}{p-1}(\log p)^{2}-4 \gamma_{0} \log p\right) \delta^{2}\right.
\end{aligned}
$$




$$
\left.+O\left(\delta^{3}\right)\right\}
$$

It is well known that $\Gamma^{\prime}(1)=-\gamma_{0}$. Let $\phi(y)=\left(\Gamma^{\prime} / \Gamma\right)(y)$. Then the formulas

$$
\phi(y)=-\gamma_{0}-\sum_{n=0}^{\infty}\left(\frac{1}{y+n}-\frac{1}{n+1}\right)
$$

and

$$
\phi^{\prime}(y)=\sum_{n=0}^{\infty} \frac{1}{(y+n)^{2}}
$$

are also well known. Putting $y=1$ in (2.6), we can see $\Gamma^{\prime \prime}(1)=\gamma_{0}^{2}+\zeta(2)$. Hence

$$
\Gamma(1+\delta)=1-\gamma_{0} \delta+\frac{1}{2}\left(\gamma_{0}^{2}+\zeta(2)\right) \delta^{2}+O\left(\delta^{3}\right),
$$

and by using this formula and (1.4) we can deduce

$$
\begin{aligned}
& 2 p^{-1-2 \delta} \Gamma(1+2 \delta) \Gamma(-\delta) \Gamma(1+\delta)^{-1} \zeta(1+2 \delta) \\
& =2 p^{-1} \delta^{-2}\left\{-\frac{1}{2}+\left(-\gamma_{0}+\log p\right) \delta+\left(2 \gamma_{0} \log p-2 \gamma_{1}-\zeta(2)-(\log p)^{2}\right) \delta^{2}\right. \\
& \left.\quad+O\left(\delta^{3}\right)\right\}
\end{aligned}
$$

Substituting (2.4) and (2.7) into the right-hand side of (2.3), and letting $\delta \rightarrow 0$, we obtain

$$
\begin{aligned}
(p-1)^{-1} V_{p} & =\zeta(2)+p^{-1}\left\{\gamma_{0}^{2}-2 \gamma_{1}-2 \zeta(2)-\frac{p}{p-1}(\log p)^{2}\right\} \\
& +2 p^{-2}\left\{\sum_{n=1}^{N-1}(-1)^{n} \zeta(1-n) \zeta(1+n) p^{1-n}+p^{1-N} R_{N}(1,1 ; p)\right\}
\end{aligned}
$$

Since $R_{N}(1,1 ; p)$ is bounded with respect to $p$, as has been shown in [5, Sect. 1], the assertion of Theorem 1 immediately follows from (2.8).

\section{The mean square at other integer points}

Let $\eta$ be a small positive constant, and define the contour $\mathscr{C}$ which consists of the half-line on the positive real axis from infinity to $\eta$, a circle of radius $\eta$ counterclockwise round the origin, and the other half-line on the positive real axis from $\eta$ to infinity. In $[5,(5.1)]$ we have shown 


$$
\begin{aligned}
R_{N}(u, v ; k)= & \frac{1}{\Gamma(u) \Gamma(v)\left(e^{2 \pi i u}-1\right)\left(e^{2 \pi i v}-1\right)} \int_{0}^{1} \frac{(1-\tau)^{N-1}}{(N-1) !} \\
& \cdot \int_{\mathscr{C}} \frac{y^{v+N-1}}{e^{y}-1} \int_{\mathscr{C}} h^{(N)}\left(x+k^{-1} \tau y\right) x^{u-1} d x d y d \tau .
\end{aligned}
$$

Since the above integral is convergent absolutely if $\Re(u)<N+1$, the identity (2.1) is valid if $\Re(u)<N+1$ and $\Re(v)<N+1$, as an identity of meromorphic functions. Hence the formula [5, (1.7)], which is obtained by putting $u=\sigma+i t$ and $v=\sigma-i t$ in (2.1), is valid in the region $\sigma<N+1$, except for the points at which some factor in the right-hand side of $[5,(1.7)]$ has a singularity.

Let $m(\geq 2)$ be an integer. If $N \geq m$, then (2.1) holds near the point $u=v$ $=m$. At the point $u=v=m$ some factors in the right-hand side of (2.1) have singularities, so we need additional calculations to obtain the asymptotic expansion of $U_{p}(m)$. Putting $u=v=m+\delta$ in (2.1), we obtain

$$
\begin{aligned}
(p & -1)^{-1} \sum_{\chi(\bmod p)}|L(m+\delta, \chi)|^{2} \\
= & \zeta(2 m+2 \delta)-p^{-2 m-2 \delta} \zeta(m+\delta)^{2} \\
& +2 p^{1-2 m-2 \delta} \Gamma(2 m-1+2 \delta) \Gamma(1-m-\delta) \Gamma(m+\delta)^{-1} \zeta(2 m-1+2 \delta) \\
& +2 p^{1-2 m-\delta}(-1)^{m-1} \cdot \frac{\Gamma(2 m-1+\delta)}{\Gamma(m) \Gamma(m+\delta)} \zeta(1+\delta) \zeta(2 m-1+\delta) \\
& +2 p^{-2 m-2 \delta} S^{*}(m+\delta ; p),
\end{aligned}
$$

where

$$
S^{*}(s ; p)=\sum_{\substack{0 \leq n \leq N-1 \\
n \neq m-1}}\left(\begin{array}{c}
-s \\
n
\end{array}\right) \zeta(s-n) \zeta(s+n) p^{s-n}+p^{s-N} R_{N}(s, s ; p) .
$$

We can see

$$
\begin{aligned}
& p^{1-2 m-2 \delta} \Gamma(2 m-1+2 \delta) \Gamma(1-m-\delta) \Gamma(m+\delta)^{-1} \zeta(2 m-1+2 \delta) \\
& \quad=p^{1-2 m}(-1)^{m} \Gamma(2 m-1) \Gamma(m)^{-2} \zeta(2 m-1) \delta^{-1} \\
& \cdot\left\{1+\left(-2 \log p+2 \phi(2 m-1)-2 \phi(m)+2 \frac{\zeta^{\prime}}{\zeta}(2 m-1)\right) \delta+O\left(\delta^{2}\right)\right\}
\end{aligned}
$$

and

$$
\begin{gathered}
p^{1-2 m-\delta}(-1)^{m-1} \frac{\Gamma(2 m-1+\delta)}{\Gamma(m) \Gamma(m+\delta)} \zeta(1+\delta) \zeta(2 m-1+\delta) \\
=p^{1-2 m}(-1)^{m-1} \Gamma(2 m-1) \Gamma(m)^{-2} \zeta(2 m-1) \delta^{-1}
\end{gathered}
$$




$$
\cdot\left\{1+\left(-\log p+\phi(2 m-1)-\phi(m)+\gamma_{0}+\frac{\zeta^{\prime}}{\zeta}(2 m-1)\right) \delta+O\left(\delta^{2}\right)\right\} .
$$

Also we note

$$
\phi(n+1)=1+\frac{1}{2}+\cdots+\frac{1}{n}-\gamma_{0}
$$

for any positive integer $n$. Substituting these relations into (3.2), and letting $\delta \rightarrow$ 0 , we obtain the assertion of Theorem 2 .

Next we proceed to the proof of Theorem 3. First we prove the following

LEMMA 1. For any integer $m \geq 0$, we have

$$
\begin{aligned}
& R_{N}(-m,-m ; k) \\
& =\left\{\begin{array}{rr}
\sum_{n=N}^{m}\left(\begin{array}{c}
m \\
n
\end{array}\right) \frac{B_{m-n+1} B_{m+n+1}}{(m-n+1)(m+n+1)} k^{N-n}+\frac{B_{2 m+2}}{2(m+1)^{2}} k^{N-m-1} & \text { if } 1 \leq N \leq m, \\
\frac{B_{2 m+2}}{2(m+1)^{2}} & \text { if } N=m+1, \\
0 & \text { if } N \geq m+2 .
\end{array}\right.
\end{aligned}
$$

Proof. Since $\Gamma(u)\left(e^{2 \pi i u}-1\right)$ tends to the value $(-1)^{m} 2 \pi i / m$ ! as $u$ tends to $-m$, from (3.1) we have

$$
\begin{aligned}
R_{N}(-m,-m ; k)= & \left(\frac{m !}{2 \pi i}\right)^{2} \int_{0}^{1} \frac{(1-\tau)^{N-1}}{(N-1) !} \int_{\mathscr{C}} \frac{y^{-m+N-1}}{e^{y}-1} \\
& \cdot \int_{\mathscr{C}} h^{(N)}\left(x+k^{-1} \tau y\right) x^{-m-1} d x d y d \tau .
\end{aligned}
$$

We can replace the integrals along $\mathscr{C}$ by the integrals along $\mathscr{C}(\eta)$, the circle of radius $\eta$ round the origin. Hence by a residue calculus, we see that the inner integral is equal to $2 \pi i h^{(N+m)}\left(k^{-1} \tau y\right) / m$ !, so

$$
R_{N}(-m,-m ; k)=\frac{m !}{2 \pi i} \int_{0}^{1} \frac{(1-\tau)^{N-1}}{(N-1) !} \int_{\mathscr{C}(\eta)} \frac{y^{-m+N-1}}{e^{y}-1} h^{(N+m)}\left(k^{-1} \tau y\right) d y d \tau
$$

Since

$$
\frac{y^{-m+N-1}}{e^{y}-1} h^{(N+m)}\left(k^{-1} \tau y\right)=\sum_{j=0}^{\infty} \frac{B_{j}}{j !} y^{j-m+N-2} \sum_{l=0}^{\infty} \frac{h^{(N+m+l)}(0)}{l !}\left(k^{-1} \tau y\right)^{l}
$$




$$
=\sum_{j=0}^{\infty} \sum_{l=0}^{\infty} \frac{B_{j} h^{(N+m+l)}(0)}{j ! l !}\left(k^{-1} \tau\right)^{l} y^{j+l-m+N-2},
$$

the inner integral in the right-hand side of (3.3) is equal to

$$
2 \pi i \sum_{j=0}^{m-N+1} \frac{B_{j} h^{(2 m-j+1)}(0)}{j !(m-N-j+1) !}\left(k^{-1} \tau\right)^{m-N-j+1} .
$$

Also we see

$$
h^{(n)}(0)=\left\{\begin{array}{cl}
\frac{1}{2} & \text { if } n=0 \\
\frac{B_{n+1}}{n+1} & \text { if } n \geq 1
\end{array}\right.
$$

because

$$
\begin{aligned}
h(z) & =\frac{1}{z} \cdot \frac{z}{e^{z}-1}+1-\frac{1}{z}=\frac{1}{z} \sum_{n=0}^{\infty} \frac{B_{n}}{n !} z^{n}+1-\frac{1}{z} \\
& =\frac{1}{2}+\sum_{n=1}^{\infty} \frac{B_{n+1}}{(n+1) !} z^{n} .
\end{aligned}
$$

Hence we have

$$
\begin{aligned}
R_{N}(-m,-m ; k)= & \frac{m !}{(N-1) !} \sum_{j=0}^{m-N+1} \frac{B_{j} B_{2 m-j+2}}{j !(m-N-j+1) !(2 m-j+2)} \\
& \cdot k^{-m+N+j-1} \int_{0}^{1}(1-\tau)^{N-1} \tau^{m-N-j+1} d \tau \\
= & m ! \sum_{j=0}^{m-N+1} \frac{B_{j} B_{2 m-j+2}}{j !(m-j+1) !(2 m-j+2)} k^{-m+N+j-1}
\end{aligned}
$$

from which the desired result follows.

Now we can deduce Theorem 3 easily by using Lemma 1 . We fix the value of $N$ arbitrarily. If we choose the value of $N \leq m+1$, then putting $u=v=-m$ $+\delta$ in (2.1), and letting $\delta \rightarrow 0$, we obtain Theorem 3 (by noticing (1.7)). If we start with the larger value of $N \geq m+2$, the following expression is suitable:

$$
\begin{aligned}
&(p-1)^{-1} \sum_{\chi(\bmod p)}|L(-m+\delta, \chi)|^{2} \\
&= \zeta(-2 m+2 \delta)-p^{2 m-2 \delta} \zeta(-m+\delta)^{2} \\
&+2 p^{1+2 m-2 \delta} \Gamma(-2 m-1+2 \delta) \Gamma(1+m-\delta) \Gamma(-m+\delta)^{-1} \zeta(-2 m-1+2 \delta) \\
&+2\left(\begin{array}{c}
m-\delta \\
m+1
\end{array}\right) \zeta(-2 m-1+\delta) \zeta(1+\delta) p^{-\delta-1}+2 p^{2 m-2 \delta} S^{* *}(-m+\delta ; p),
\end{aligned}
$$


where

$$
S^{* *}(s ; p)=\sum_{\substack{0 \leq n \leq N-1 \\
n \neq m+1}}\left(\begin{array}{c}
-s \\
n
\end{array}\right) \zeta(s-n) \zeta(s+n) p^{s-n}+p^{s-N} R_{N}(s, s ; p) .
$$

Letting $\delta \rightarrow 0$ in the above and using Lemma 1 , we obtain the same conclusion of Theorem 3 .

\section{Supplementary proofs}

In this section we describe two supplementary arguments. We first show a direct elementary proof of (1.5). Since

$$
C^{\prime}(y)=\sum_{n=1}^{\infty} \frac{1}{(n+y)^{2}}=\phi^{\prime}(y)-\frac{1}{y^{2}}
$$

(see (2.6)) and $C(1)=1$, we have

$$
C(y)=\phi(y)+\frac{1}{y}+\gamma_{0} .
$$

Hence, with (2.5) it follows that

$$
C(y)=1-\sum_{n=1}^{\infty}\left(\frac{1}{y+n}-\frac{1}{n+1}\right)
$$

so

$$
\int_{0}^{1} C(y)^{2} d y=1-2 \sum_{n=1}^{\infty} \int_{0}^{1}\left(\frac{1}{y+n}-\frac{1}{n+1}\right) d y+\int_{0}^{1}\left(\sum_{n=1}^{\infty}\left(\frac{1}{y+n}-\frac{1}{n+1}\right)\right)^{2} d y
$$

Using the formula

$$
\sum_{k=1}^{N} \frac{1}{k}=\log N+\gamma_{0}+O\left(N^{-1}\right)
$$

we have

$$
\sum_{n=1}^{\infty} \int_{0}^{1}\left(\frac{1}{y+n}-\frac{1}{n+1}\right) d y=\lim _{N \rightarrow \infty}\left(\log (N+1)-\sum_{n=1}^{N} \frac{1}{n+1}\right)=1-\gamma_{0},
$$

hence

$$
\int_{0}^{1} C(y)^{2} d y=-1+2 \gamma_{0}+\int_{0}^{1}\left(\sum_{n=1}^{\infty}\left(\frac{1}{y+n}-\frac{1}{n+1}\right)\right)^{2} d y
$$




$$
=-1+2 \gamma_{0}+\lim _{N \rightarrow \infty} \Sigma_{1}(N)
$$

where

$\sum_{1}(N)$

$$
\begin{aligned}
&= \sum_{m, n \leq N}\left\{\int_{0}^{1} \frac{d y}{(y+m)(y+n)}-\frac{2}{m+1} \int_{0}^{1} \frac{d y}{y+n}+\frac{1}{(m+1)(n+1)}\right\} \\
&=1-\frac{1}{N+1}+\sum_{\substack{m, n \leq N \\
m \neq n}} \frac{1}{n-m} \log \frac{n(1+m)}{m(1+n)} \\
&-2 \sum_{m \leq N} \frac{\log (N+1)}{m+1}+\left(\sum_{n \leq N} \frac{1}{n+1}\right)^{2} .
\end{aligned}
$$

Applying (4.1) to the last two terms of the above, we obtain

$$
\Sigma_{1}(N)=1+\left(1-\gamma_{0}\right)^{2}+2 \sum_{2}(N)-(\log (N+1))^{2}+O\left(\frac{\log N}{N}\right),
$$

where

$$
\begin{aligned}
\sum_{2}(N) & =\sum_{m<n \leq N} \frac{1}{n-m} \log \frac{n(1+m)}{m(1+n)} \\
& =\sum_{n \leq N} \log \frac{n}{1+n} \sum_{k=1}^{n-1} \frac{1}{k}+\sum_{m<N} \log \frac{1+m}{m} \sum_{k=1}^{N-m} \frac{1}{k} \\
& =\sum_{3}(N)+\sum_{4}(N), \text { say. }
\end{aligned}
$$

We see, by using (4.1),

$$
\begin{aligned}
\sum_{3}(N) & =\sum_{k \leq N-1} \frac{1}{k} \sum_{k+1 \leq n \leq N}\{\log n-\log (1+n)\} \\
& =\sum_{k \leq N-1} \frac{\log (k+1)}{k}-\log (N+1) \sum_{k \leq N-1} \frac{1}{k} \\
& =\sum_{k \leq N} \frac{\log (k+1)}{k}-(\log N)^{2}-\gamma_{0} \log N+O\left(\frac{\log N}{N}\right)
\end{aligned}
$$

and

$$
\begin{aligned}
\sum_{4}(N) & =\sum_{k \leq N-1} \frac{1}{k} \sum_{m \leq N-k}\{\log (1+m)-\log m\} \\
& =\sum_{k \leq N-1} \frac{\log N}{k}+\sum_{k \leq N-1} \frac{1}{k} \log \left(1-\frac{k-1}{N}\right)
\end{aligned}
$$




$$
=(\log N)^{2}+\gamma_{0} \log N+\sum_{k \leq N-1} \frac{1}{k} \log \left(1-\frac{k}{N}\right)+O\left(\frac{\log N}{N}\right)
$$

so

$$
\sum_{2}(N)=\sum_{k \leq N} \frac{\log (k+1)}{k}+\sum_{k \leq N-1} \frac{1}{k} \log \left(1-\frac{k}{N}\right)+O\left(\frac{\log N}{N}\right) .
$$

Also we have

$$
A=\sum_{k=1}^{\infty} \frac{\log (k+1)}{k(k+1)}=\lim _{N \rightarrow \infty}\left\{\sum_{k \leq N} \frac{\log (k+1)}{k}-\sum_{k \leq N} \frac{\log (k+1)}{k+1}\right\},
$$

hence, combining with (4.2), (4.3) and (4.4), we obtain

$$
\begin{aligned}
1+ & 2 A-\int_{0}^{1} C(y)^{2} d y \\
& =-\gamma_{0}^{2}+\lim _{N \rightarrow \infty}\left\{(\log (N+1))^{2}-2 \sum_{k \leq N+1} \frac{\log k}{k}-2 \sum_{k \leq N-1} \frac{1}{k} \log \left(1-\frac{k}{N}\right)\right\} .
\end{aligned}
$$

Since

$$
\gamma_{1}=\lim _{N \rightarrow \infty}\left\{\frac{1}{2}(\log (N+1))^{2}-\sum_{k \leq N+1} \frac{\log k}{k}\right\}
$$

(see e.g., Theorem 1.3 in Ivić [4]), we have

$$
\begin{aligned}
1+2 A-\int_{0}^{1} C(y)^{2} d y & =-\gamma_{0}^{2}+2 \gamma_{1}-2 \lim _{N \rightarrow \infty} \sum_{k \leq N-1} \frac{1}{k} \log \left(1-\frac{k}{N}\right) \\
& =-\gamma_{0}^{2}+2 \gamma_{1}-2 \int_{0}^{1} \frac{\log (1-x)}{x} d x \\
& =-\gamma_{0}^{2}+2 \gamma_{1}-2 \int_{0}^{\infty} \frac{u}{1-e^{u}} d u \\
& =-\gamma_{0}^{2}+2 \gamma_{1}+2 \zeta(2),
\end{aligned}
$$

which is the desired result.

Next we show how we can deduce Theorem 3 from (1.10). Define

$$
F(z, w)=\sum_{a=1}^{p-1} f\left(\frac{a}{p}, z\right) f\left(\frac{a}{p}, w\right)
$$

for $|z|<2 \pi,|w|<2 \pi$. Using the expression (1.8), we have

$$
F(z, w)=z w\left(\exp \left(\frac{z+w}{p}\right)-1\right)^{-1}+z w\left(e^{z}-1\right)^{-1}\left(\exp \left(\frac{z+w}{p}\right)-1\right)^{-1}
$$




$$
+z w\left(e^{w}-1\right)^{-1}\left(\exp \left(\frac{z+w}{p}\right)-1\right)^{-1}-z w\left(e^{z}-1\right)^{-1}\left(e^{w}-1\right)^{-1}
$$

and, using the relation (1.6) in the right-hand side of the above, we obtain

$$
\begin{aligned}
& F\left(e^{i \theta} z, e^{-i \theta} z\right) \\
& =z\left(\frac{2 \cos \theta}{p}\right)^{-1} \sum_{j=0}^{\infty} \frac{B_{j}}{j !}\left(\frac{2 \cos \theta}{p} z\right)^{j} \\
& \quad+\left(e^{-i \theta}\right)^{-1}\left(\frac{2 \cos \theta}{p}\right)^{-1} \sum_{k=0}^{\infty} \frac{B_{k}}{k !}\left(e^{-i \theta} z\right)^{k} \sum_{h=0}^{\infty} \frac{B_{h}}{h !}\left(\frac{2 \cos \theta}{p} z\right)^{h} \\
& \quad+\left(e^{i \theta}\right)^{-1}\left(\frac{2 \cos \theta}{p}\right)^{-1} \sum_{k=0}^{\infty} \frac{B_{k}}{k !}\left(e^{i \theta} z\right)^{k} \sum_{h=0}^{\infty} \frac{B_{h}}{h !}\left(\frac{2 \cos \theta}{p} z\right)^{h} \\
& \quad-\sum_{k=0}^{\infty} \frac{B_{k}}{k !}\left(e^{i \theta} z\right)^{k} \sum_{h=0}^{\infty} \frac{B_{h}}{h !}\left(e^{-i \theta} z\right)^{h} \\
& =\sum_{j=1}^{\infty} \frac{B_{j-1}}{(j-1) !}\left(\frac{2 \cos \theta}{p}\right)^{j-2} z^{j} \\
& \quad+\sum_{j=0}^{\infty} z^{j} \sum_{h=0}^{j} \frac{B_{j-h} B_{h}}{(j-h) ! h !}\left\{2 \cos ((j-h-1) \theta)\left(\frac{2 \cos \theta}{p}\right)^{h-1}-e^{i(j-2 h) \theta}\right\} .
\end{aligned}
$$

On the other hand, from (1.9) and (4.5), we have

$$
\frac{1}{2 \pi} \int_{0}^{2 \pi} F\left(e^{i \theta} z, e^{-i \theta} z\right) d \theta=\sum_{k=0}^{\infty}\left(\sum_{a=1}^{p-1} B_{k}(a / p)^{2}\right) \frac{z^{2 k}}{(k !)^{2}} .
$$

Therefore, $((m+1) !)^{-2} \sum_{a=1}^{p-1} B_{m+1}(a / p)^{2}$ coincides with the coefficient of $z^{2 m+2}$ in the left-hand side of (4.7), which is, by using the expression (4.6), equal to

$$
\begin{aligned}
& \frac{B_{2 m+1}}{(2 m+1) !} 2^{2 m} p^{-2 m}(2 \pi)^{-1} \int_{0}^{2 \pi} \cos ^{2 m} \theta d \theta \\
& +\sum_{h=0}^{2 m+2} \frac{B_{2 m+2-h} B_{h}}{(2 m+2-h) ! h !}(2 \pi)^{-1}\left\{2^{h} p^{1-h} \int_{0}^{2 \pi} \cos ((2 m+1-h) \theta) \cos ^{h-1} \theta d \theta\right. \\
& \left.\quad-\int_{0}^{2 \pi} e^{i(2 m+2-2 h) \theta} d \theta\right\} .
\end{aligned}
$$

If $\alpha+\beta>1$, then the formula

$$
\int_{0}^{\pi / 2} \cos ((\alpha-\beta) \theta) \cos ^{\alpha+\beta-2} \theta d \theta=\frac{\pi}{(\alpha+\beta-1) 2^{\alpha+\beta-1} B(\alpha, \beta)}
$$

holds, where $B($,$) denotes the beta-function (Whittaker-Watson [14], Chapter$ 
XII, Miscellaneous Examples 39, p.263). Hence

$$
\frac{1}{2 \pi} \int_{0}^{2 \pi} \cos ((2 m+1-h) \theta) \cos ^{h-1} \theta d \theta=\frac{(h-1) \cdots(h-m)}{2^{h-1} m !}
$$

for any $h \geq 1$. Therefore we now obtain

$$
\begin{aligned}
& ((m+1) !)^{-2} \sum_{a=1}^{p-1} B_{m+1}(a / p)^{2} \\
& =\frac{B_{2 m+1}}{(m !)^{2}(2 m+1)} p^{-2 m} \\
& \quad+2 \sum_{k=0}^{m+1} \frac{B_{m+1-k} B_{m+1+k}}{(m+1-k) !(m+1+k) !} \cdot \frac{(k+1) \cdots(k+m)}{m !} p^{-m-k} \\
& \quad+(-1)^{m} \frac{B_{2 m+2}}{(2 m+2) !} p-\frac{B_{m+1}^{2}}{((m+1) !)^{2}}
\end{aligned}
$$

From (1.10) it follows that

$$
U_{p}(-m)=p^{2 m}(m+1)^{-2} \sum_{a=1}^{p-1} B_{m+1}(a / p)^{2} .
$$

Substituting (4.8) into the right-hand side of the above, and using (1.7), we arrive at the assertion of Theorem 3 .

\section{The composite case}

In this section $q$ denotes an arbitrary positive number $(\geq 2)$, and by $\mu(n)$ we mean the Möbius function. In [5], the following formula is implicitly included.

Lemma 2. For any $N \geq 1$, we have

$$
\begin{aligned}
& \varphi(q)^{-1} \sum_{\substack{\chi(\bmod q) \\
\chi \neq \chi_{0}}} L(u, \chi) L(v, \bar{\chi}) \\
& =-\varphi(q)^{-1} L\left(u, \chi_{0}\right) L\left(v, \chi_{0}\right)+\zeta(u+v) \prod_{p \mid q}\left(1-p^{-u-v}\right) \\
& \quad+q^{-u-v} \varphi(q) \Gamma(u+v-1) \zeta(u+v-1)\left(\frac{\Gamma(1-v)}{\Gamma(u)}+\frac{\Gamma(1-u)}{\Gamma(v)}\right) \\
& \quad+q^{-u-v} \sum_{k \mid q} \mu(q / k)(S(u, v ; k)+S(v, u ; k))
\end{aligned}
$$

where $k$ runs over all positive divisors of $q$, $p$ runs over all prime divisors of $q$, and 


$$
S(u, v ; k)=\sum_{n=0}^{N-1}\left(\begin{array}{c}
-v \\
n
\end{array}\right) \zeta(u-n) \zeta(v+n) k^{u-n}+k^{u-N} R_{N}(u, v ; k) .
$$

In fact, applying the same method as that developed in [5, Sect. 4] to the each term in the second sum of the right-hand side of $[5,(2.7)]$, and combining with $[5$, (2.2)], we obtain Lemma 2. We have used Lemma 2 in the proof of [5, (1.3) and (1.4)], the details being omitted in [5]. (Here we mention the existence of the related articles of Balasubramanian [3] and Zhang [15] [16], which we have missed in the references of [5].)

Let $P(s)=\Pi_{p \mid q}\left(1-p^{-s}\right)$. Then, noting $\varphi(q)=q P(1)$ and

$$
\sum_{k \mid q} \mu(q / k) k^{s}=q^{s} P(s)
$$

from Lemma 2 we have

$$
\begin{aligned}
& \varphi(q)^{-1} \sum_{\substack{x(\mathrm{mod} q) \\
\chi \neq x_{0}}}|L(1+\delta, \chi)|^{2} \\
& =-q^{-1} P(1)^{-1} P(1+\delta)^{2} \zeta(1+\delta)^{2}+\zeta(2+2 \delta) P(2+2 \delta) \\
& +2 q^{-1-2 \delta} P(1) \Gamma(1+2 \delta) \frac{\Gamma(-\delta)}{\Gamma(1+\delta)} \zeta(1+2 \delta)+2 q^{-1-\delta} P(1+\delta) \zeta(1+\delta)^{2} \\
& \quad+2 q^{-2-2 \delta} \sum_{k \mid q} \mu(q / k)\left\{\sum_{n=1}^{N-1}\left(\begin{array}{c}
-1-\delta \\
n
\end{array}\right) \zeta(1+\delta-n) \zeta(1+\delta+n) k^{1+\delta-n}\right. \\
& \left.\quad+k^{1+\delta-N} R_{N}(1+\delta, 1+\delta ; k)\right\} .
\end{aligned}
$$

We take the limit $\delta \rightarrow 0$ as in Section 2. Since

$$
\begin{aligned}
& q^{-1} P(1)^{-1} P(1+\delta)^{2} \zeta(1+\delta)^{2} \\
& =q^{-1} P(1)^{-1}\left\{P(1)^{2} \delta^{-2}+\left(2 P(1)^{2} \gamma_{0}+2 P(1) P^{\prime}(1)\right) \delta^{-1}\right. \\
& \left.\quad+P(1)^{2}\left(\gamma_{0}^{2}+2 \gamma_{1}\right)+4 P(1) P^{\prime}(1) \gamma_{0}+P^{\prime}(1)^{2}+P(1) P^{\prime \prime}(1)+O(\delta)\right\} \\
& 2 q^{-1-2 \delta} P(1) \Gamma(1+2 \delta) \Gamma(-\delta) \Gamma(1+\delta)^{-1} \zeta(1+2 \delta) \\
& =2 q^{-1} P(1)\left\{-\frac{1}{2} \delta^{-2}+\left(-\gamma_{0}+\log q\right) \delta^{-1}-2 \gamma_{1}-\zeta(2)+2 \gamma_{0} \log q\right. \\
& \left.\quad-(\log q)^{2}+O(\delta)\right\}
\end{aligned}
$$

and

$$
\begin{aligned}
& 2 q^{-1-\delta} P(1+\delta) \zeta(1+\delta)^{2} \\
& =2 q^{-1}\left\{P(1) \delta^{-2}+\left(P(1)\left(2 \gamma_{0}-\log q\right)+P^{\prime}(1)\right) \delta^{-1}\right.
\end{aligned}
$$




$$
\begin{aligned}
& +P(1)\left(\gamma_{0}^{2}+2 \gamma_{1}-2 \gamma_{0} \log q+\frac{1}{2}(\log q)^{2}\right) \\
& \left.+P^{\prime}(1)\left(2 \gamma_{0}-\log q\right)+\frac{1}{2} P^{\prime \prime}(1)+O(\delta)\right\},
\end{aligned}
$$

letting $\delta \rightarrow 0$ in (5.2) we have

$$
\begin{aligned}
& \varphi(q)^{-1} V_{q} \\
&=q^{-1} P(1)\left\{\gamma_{0}^{2}-2 \gamma_{1}-(\log q)^{2}-2 \zeta(2)-2 \frac{P^{\prime}}{P}(1) \log q-\left(\frac{P^{\prime}}{P}(1)\right)^{2}\right\} \\
&+\zeta(2) P(2)+2 q^{-2} \sum_{k \mid q} \mu(q / k)\left\{\sum_{n=1}^{N-1}(-1)^{n} \zeta(1-n) \zeta(1+n) k^{1-n}+k^{1-N} R_{N}(1,1 ; k)\right\} .
\end{aligned}
$$

Hence, noting

$$
\frac{P^{\prime}}{P}(1)=\sum_{p \mid q} \frac{\log p}{p-1}
$$

we now obtain

THEOREM 4. For any integer $N \geq 1$, we have

$$
\begin{aligned}
\varphi(q)^{-1} V_{q}= & q^{-1} \prod_{p \mid q}\left(1-p^{-1}\right)\left\{\gamma_{0}^{2}-2 \gamma_{1}-(\log q)^{2}-2 \zeta(2)-2(\log q) \sum_{p \mid q} \frac{\log p}{p-1}\right. \\
& \left.-\left(\sum_{p \mid q} \frac{\log p}{p-1}\right)^{2}\right\}+\zeta(2) \prod_{p \mid q}\left(1-p^{-2}\right) \\
& \quad+2 q^{-2} \sum_{k \mid q} k \mu(q / k)\left\{\sum_{n=1}^{N-1}(-1)^{n} \zeta(1-n) \zeta(1+n) k^{-n}+O\left(k^{-N}\right)\right\} .
\end{aligned}
$$

This is a refinement of Zhang's formula [18], which asserts

$$
\begin{aligned}
\varphi(q)^{-1} V_{q}= & -q^{-2} \varphi(q)\left\{1+2 A-\int_{0}^{1} C^{2}(y) d y+\left(\log q+\sum_{p \mid q} \frac{\log p}{p-1}\right)^{2}\right\} \\
& +\zeta(2) \prod_{p \mid q}\left(1-\frac{1}{p^{2}}\right)+O\left(\frac{1}{\varphi(q) \log q}\right)
\end{aligned}
$$

(see the Remark in Section 1). We should note that (5.3) is not the "asymptotic expansion" in the strict sense. In particular, the sum with respect to $k$ in the right-hand side of (5.3) includes the term corresponding to $k=1$, with the error $O(1)$. But this term can be calculated explicitly, and the errors corresponding to $k$ $>1$ are decreasing as $N \rightarrow \infty$. Hence, for instance in case $q=p^{a}$ is a prime power, we can deduce the asymptotic expansion of $V_{q}$ with respect to $p$. 
Lemma 2 is valid, as an identity of meromorphic functions, in the region $\{(u, v) \mid \Re(u)<N+1, \Re(v)<N+1\}$, by (3.1). Hence the formula [5, (1.3)] is valid in the region $\sigma<N+1$, except for the points at which some factor in the right-hand side of $[5,(1.3)]$ has a singularity. As for the exceptional points, we can show generalizations of Theorems 2 and 3 to the case of general modulus. We conclude this paper with the statements of these results. The proofs are simple generalizations of the argument in Section 3, and are omitted. (Theorem 6 can also be deduced from (1.10).)

THEOREM 5. For any integers $m$ and $N$ satisfying $2 \leq m \leq N$, we have

$$
\begin{aligned}
U_{q}(m)= & \zeta(2 m) \prod_{p \mid q}\left(1-p^{-2 m}\right)+2 q^{-2 m} \varphi(q)(-1)^{m} \frac{(2 m-2) !}{((m-1) !)^{2}} \zeta(2 m-1) \\
& \cdot\left\{-\log q-\sum_{p \mid q} \frac{\log p}{p-1}+\sum_{h=m}^{2 m-2} \frac{1}{h}-\gamma_{0}+\frac{\zeta^{\prime}}{\zeta}(2 m-1)\right\} \\
& +2 q^{-2 m} \sum_{k \mid q} \mu(q / k) S^{*}(m ; k) .
\end{aligned}
$$

THEOREM 6. For any integer $m \geq 0$, we have the following finite expression of $U_{q}(-m)$ :

$$
\begin{aligned}
U_{q}(-m)= & (-1)^{m-1} q^{2 m} \varphi(q) \frac{(m !)^{2}}{(2 m+1) !} \zeta(-2 m-1) \\
& +2 q^{2 m} \sum_{k \mid q} \mu(q / k) \sum_{n=0}^{m}\left(\begin{array}{c}
m \\
n
\end{array}\right) \zeta(-m-n) \zeta(-m+n) k^{-m-n} \\
& +q^{-1} \frac{B_{2 m+2}}{(m+1)^{2}} \prod_{p \mid q}\left(1-p^{2 m+1}\right) .
\end{aligned}
$$

\section{Appendix}

Here we give the proof of (1.13). Satz 3 of Zimmert [19] asserts, in our case, that

$$
R_{p} \geq 2 c(\alpha) p(g(\alpha))^{(p-1) / 2}
$$

for any $\alpha>0$, where

$$
c(\alpha)=\frac{1}{2}(1+\alpha)(1+2 \alpha) \exp \left(-3-2 \alpha^{-1}\right)
$$


and

$$
g(\alpha)=(2 \sqrt{\pi})^{-1} \Gamma(1+\alpha) \Gamma\left(\frac{3}{2}+\alpha\right) \exp \left\{-(1+\alpha)\left(\phi\left(\frac{1+\alpha}{2}\right)+\phi\left(1+\frac{\alpha}{2}\right)\right)\right\}
$$

Using (2.5) and (2.6), we see that

$$
\begin{aligned}
& \frac{d}{d \alpha} \log g(\alpha) \\
& =-\left(\frac{1+\alpha}{2}\right)^{2} \sum_{n=0}^{\infty}\left\{\frac{1}{\left(\frac{1+\alpha}{2}+n\right)^{2}(1+\alpha+n)}+\frac{1}{\left(1+\frac{\alpha}{2}+n\right)^{2}\left(\frac{3}{2}+\alpha+n\right)}\right\},
\end{aligned}
$$

which is negative for $\alpha \geq 0$, hence $g(\alpha)$ is monotonically decreasing in the same range of $\alpha$. Since $g(0)=e^{2 \gamma_{0}}$, we have

$$
R_{p} \geq 2 K(\varepsilon) p\left(e^{2 \gamma_{0}}-\varepsilon\right)^{(p-1) / 2}
$$

for any $\varepsilon>0$, with the constant $K(\varepsilon)$ depending only on $\varepsilon$.

Combining (1.11) with (3), (4), (5) and (13) in Wang [12], we get

$$
h_{p} R_{p} \leq 2 p^{\frac{p}{2}}\left(\frac{p-1}{8 p\left(2^{\nu}-1\right)^{4 / \nu}}\right)^{\frac{p-1}{4}}\left(\frac{4(p-1) S-2(\log p)^{2}}{p-3}\right)^{\frac{p-3}{4}},
$$

where $\nu$ is the order of $2 \bmod p$, and

$$
S=\sum_{r=1}^{(p-2) / 2}\left(\log \tan \frac{\pi r}{p}\right)^{2}
$$

Wang proved an asymptotic formula of $S$ ([12, formula (11)]). However, to obtain an upperbound of $h_{p}$, it is enough to use the inequality

$$
S \leq \frac{\pi^{2}}{8} p-\frac{1}{2}(\log p)^{2}+\left(\frac{1}{2}+\frac{2 \sqrt{3}}{3}\right) \log p .
$$

To prove his asymptotic formula, Wang divided $S=S_{1}+S_{2}+S_{3}+S_{4}+S_{5}$. As for $S_{1}$ and $S_{5}$, we use Wang's result $S_{1}=\frac{\pi^{2}}{8} p$ and $S_{5} \leq \frac{2 \sqrt{3}}{3} \log p$. Wang also proved

$$
S_{2}+S_{3}=-\frac{1}{2}\left(\log \cot \frac{\pi}{2 p}\right)^{2}+R
$$

with a certain remainder integral $R$. Instead of Wang's estimates of $S_{4}$ and $R$, we use the simple facts $S_{4} \leq 0$ and $R \leq 0$. Then we obtain (A.4). Hence the proof of 
(A.4) is simpler than that of Wang's asymptotic formula, and actually it gives better results. Applying (A.2) and (A.4) to (A.3), we obtain

$$
h_{p} \leq \frac{1}{K(\varepsilon)}\left(\frac{\left(e^{2 \gamma_{0}}-\varepsilon\right)^{-2}(p-3)}{4 \pi^{2} p\left(2^{\nu}-1\right)^{4 / \nu}\left(1-X_{p}\right)}\right)^{\frac{1}{4}}\left(\frac{\left(e^{2 \gamma_{0}}-\varepsilon\right)^{-2} \pi^{2} p^{2} Y_{p}}{16}\right)^{\frac{p-2}{4}},
$$

where

$$
X_{p}=\frac{4(\log p)^{2}}{\pi^{2}(p-1)}-\frac{8}{\pi^{2}}\left(\frac{1}{2}+\frac{2 \sqrt{3}}{3}\right) \frac{\log p}{p}
$$

and

$$
Y_{p}=\frac{\left(1-p^{-1}\right)^{2}\left(1-X_{p}\right)}{\left(2^{\nu}-1\right)^{4 / \nu}\left(1-3 p^{-1}\right)} .
$$

Since $\nu$ tends to infinity as $p$ tends to infinity, and

$$
\lim _{\nu \rightarrow \infty}\left(2^{\nu}-1\right)^{4 / \nu}=16,
$$

it follows that for any $\varepsilon>0$, there exists a sufficiently large $p_{0}=p_{0}(\varepsilon)$, for which the inequalities

$$
\begin{gathered}
\left(2^{\nu}-1\right)^{4 / \nu} \geq 16(1-\varepsilon) \\
1-X(p) \geq 1-\varepsilon
\end{gathered}
$$

and

$$
\begin{aligned}
\frac{p-2}{4} \log Y_{p} & \leq-(p-2) \log 2-\frac{p-2}{4} \log X_{p}+\frac{1}{4}+\varepsilon \\
& \leq-(p-2) \log 2-\frac{1-\varepsilon}{\pi^{2}}(\log p)^{2}+\frac{1}{4}+2 \varepsilon
\end{aligned}
$$

hold for any $p \geq p_{0}$. Substituting these inequalities into (A.5), we obtain

$$
h_{p} \leq \frac{1}{K(\varepsilon)}\left(\frac{\left(e^{2 \gamma_{0}}-\varepsilon\right)^{-2} e(1+\varepsilon)}{64 \pi^{2}}\right)^{\frac{1}{4}}\left(\frac{\left(e^{2 \gamma_{0}}-\varepsilon\right)^{-1} \pi}{16} p\right)^{\frac{p-2}{2}} \exp \left(-\frac{1-\varepsilon}{\pi^{2}}(\log p)^{2}\right)
$$

(here, $\varepsilon$ 's are not necessarily the same in each occurrence). Since $16 e^{2 \gamma_{0}}=$ $50.7549 \cdots \cdots>50$, taking a sufficiently small value of $\alpha$, we obtain (1.13).

Remark. The inequality (A.2) can be generalized to arbitrary number fields. Let $F$ be an algebraic number field, $R_{F}$ the regulator of $F, w_{F}$ the number of the roots of unity included in $F, r_{1}$ (resp. $2 r_{2}$ ) the number of real (resp. complex) embeddings of $F$ into the complex number field. Zimmert's theorem can be stated 
as

$$
R_{F} / w_{F} \geq c(\alpha) f(\alpha)^{r_{1}} g(\alpha)^{r_{2}}
$$

where

$$
f(\alpha)=\frac{1}{2} \Gamma(1+\alpha) \exp \left(-(1+\alpha) \phi\left(\frac{1+\alpha}{2}\right)\right)
$$

and $c(\alpha), g(\alpha)$ are as above. By choosing $\alpha=1$ in (A.6), we have $R_{F} / w_{F} \geq$ $0.08 \exp \left(0.46 r_{1}+0.1 r_{2}\right)$. This is stated by Zimmert himself, and used by both Slavutskir [7] [8] and Wang [12]. Slavutskir [9] considered the case $\alpha=1 / 2$, and proved $R_{F} / w_{F} \geq 0.00136 \exp \left(0.81 r_{1}+0.57 r_{2}\right)$. We have already proved in the above that $g(\alpha)$ is monotonically decreasing. It is also true for $f(\alpha)$, because

$$
\frac{d}{d \alpha} \log f(\alpha)=-\left(\frac{1+\alpha}{2}\right)^{2} \sum_{n=0}^{\infty} \frac{1}{\left(\frac{1+\alpha}{2}+n\right)^{2}(1+\alpha+n)} .
$$

Therefore, noting $\log f(0)=\log 2-\gamma_{0}$, for any $\varepsilon>0$ we obtain

$$
R_{F} / w_{F} \geq K(\varepsilon) \exp \left(\left(\log 2+\gamma_{0}-\varepsilon\right) r_{1}+\left(2 \gamma_{0}-\varepsilon\right) r_{2}\right) .
$$

We note $\log 2+\gamma_{0}=1.2703 \cdots$ and $2 \gamma_{0}=1.1544 \cdots$. Though $K(\varepsilon)(=c(\alpha))$ tends to 0 as $\varepsilon \rightarrow 0$, the coefficients of $r_{1}$ and $r_{2}$ are more important when we consider the situation at least one of $r_{1}$ and $r_{2}$ is large.

\section{REFERENCES}

[1] Ankeny, N. C. and Chowla, S., The class number of the cyclotomic field, Canad. J. Math., 3 (1951), 486-494.

[2] Atkinson, F. V., The mean-value of the Riemann zeta function, Acta Math., 81 (1949), 353-376.

[3] Balasubramanian, R., A note on Dirichlet's L-functions, Acta Arith., 38 (1980), $273-283$.

[4] Ivić, A., The Riemann zeta-function. The theory of the Riemann zeta-function with applications, Wiley-Interscience, 1985.

[5] Katsurada, M. and Matsumoto, K., Asymptotic expansions of the mean values of Dirichlet $L$-functions, Math. Z., 208 (1991), 23-39.

[6] Motohashi, Y., A note on the mean value of the zeta and $L$-functions I, Proc. Japan Acad. Ser. A, 61 (1985), 222-224.

[ 7 ] Slavutskiř, I. Sh., Mean value of $\mathscr{L}$-functions and the ideal class number of a cyclotomic field, in "Algebraic systems with one action and relation", Leningrad. Gos. Ped. Inst., Leningrad, 1985, pp.122-129. (Russian)

[ 8 ] - Mean value of $L$-functions and the class number of a cyclotomic field, $Z$ ap. Nauchn. Sem. LOMI, 154 (1986), 136-143 (Russian) = J. Soviet Math., 43 (1988), 
$2596-2601$.

[9] - The estimate for the regulator of algebraic fields, Math. Slovaca, 41 (1991), 311-314. (Russian)

[10] Titchmarsh, E. C., The theory of the Riemann zeta-function, Oxford, 1951.

[11] Walum, H., An exact formula for an average of $L$-series, Illinois J. Math., 26 (1982), 1-3.

[12] Wang, Lan, An upper bound of class number of cyclotomic field $\mathbf{Q}\left(\zeta_{p}\right)$, Chinese Ann. Math., 12B (1991), 90-95.

[13] Washington, L. C., Introduction to cyclotomic fields, Springer, 1982.

[14] Whittaker, E. T. and Watson, G. N., A course of modern analysis, 4-th ed., Cambridge Univ. Press, 1927.

[15] Zhang, Wenpeng, On the mean square value of Dirichlet $L$-functions, Adv. in Math. (China), 19 (1990), 321-333. (Chinese. English summary)

[16] - On the mean square value formulas of $L$-functions, Chinese Ann. Math. Ser. A, 11 (1990), 121-127. (Chinese)

[17] - On the mean value of $L$-functions, J. Math. Res. Exposition, 10 (1990), 355-360. (Chinese. English summary)

[18] - On an elementary result of $L$-functions, Adv. in Math. (China), 19 (1990), 478-487. (Chinese. English summary)

[19] Zimmert, R., Ideale kleiner Norm in Idealklassen und eine Regulatorabschätzung, Invent. Math., 62 (1981), 367-380.

M. Katsurada

Department of Mathematics

Faculty of Science

Kagoshima University, Korimoto

Kagoshima 890, Japan

K. Matsumoto

Department of Mathematics

Faculty of Education

Iwate University

Ueda, Morioka 020, Japan 